\title{
Morphological Assessment of TMJ Spaces, Mandibular Condyle, and Glenoid Fossa Using Cone Beam Computed Tomography (CBCT): A Retrospective Analysis
}

\author{
Nithin $^{1} \quad$ Junaid Ahmed ${ }^{1} \quad$ Nanditha Sujir ${ }^{1} \quad$ Nandita Shenoy $^{1} \quad$ Almas Binnal $^{1} \quad$ Ravikiran Ongole $^{1}$
}

${ }^{1}$ Department of Oral Medicine and Radiology, Manipal College of Dental Sciences, Mangalore, Manipal Academy of Higher Education, Manipal, Karnataka, India

Indian J Radiol Imaging 2021;31:78-85.

\begin{abstract}
Address for correspondence: Dr Junaid Ahmed, MDS, Department of Oral Medicine and Radiology, Manipal College of Dental Sciences, Mangalore, Manipal Academy of Higher Education, Manipal, Karnataka - 576104, India (e-mail: junaid.ahmed@manipal.edu).
\end{abstract}

\begin{abstract}
Keywords

- cone beam CT

- diagnostic

- glenoid fossa

- imaging

- tomography

- temporomandibular joint

Background The temporomandibular joint (TMJ) is included in the category of ginglymoarthrodial synovial joints. The mandibular condyle plays a vital part in the development of the craniofacial complex. Hence, the evaluation and assessment of the condylar volume and its morphology are of utmost importance.

Aim The aim of this research was to use cone beam computed tomography (CBCT) imaging modality to evaluate the morphology of the mandibular condyle and glenoid fossa in a selected population and document any morphometric changes.

Setting and Design It is an observational study.

Materials and Methods A retrospective CBCT analysis was performed on 119 patients. The length, width, height, linear measurements of the joint spaces (anterior, posterior, and superior), volume of the condyle, and roof of glenoid fossa thickness were evaluated on both the left and right sides in both males and females.

Results The height, width, and length of mandibular condyle were significantly increased in males on both sides. Except for the left anterior space, all other spaces were significantly larger in males. Overall, the volume of the condyles in males was significantly increased on both sides.

Conclusion CBCT can be a valuable diagnostic aid in the evaluation of various dimensions, joint spaces, and condylar volume in different planes and thus, can be a useful predictor in the assessment of treatment outcomes of disorders affecting the TMJ.
\end{abstract}

\section{Introduction}

The temporomandibular joint (TMJ) is included in the category of ginglymoarthrodial synovial joint and consists of an articular disk, fibrous capsule, articular eminence, synovial membrane, synovial fluid, and associated ligaments. ${ }^{1}$ The mandibular condyle forms the inferior part of the articular

published online May 31, 2021
DOI https://doi.org/

$10.1055 / \mathrm{s}-0041-1729488$

ISSN 0971-3026 surface of the TMJ, whereas the superior part of the articular surface is formed by the articular eminence of the temporal bone and the mandibular fossa (also known as glenoid fossa). ${ }^{2}$

The oral cavity, teeth, and the associated muscles of mastication have a very close relationship with the TMJ. Together with the masticatory muscles, the oral structures regulate the position and function of the TMJ. All associated structures

(c) 2021. Indian Radiological Association

This is an open access article published by Thieme under the terms of the Creative Commons Attribution-NonDerivative-NonCommercial-License, permitting copying and reproduction so long as the original work is given appropriate credit. Contents may not be used for commercial purposes, or adapted, remixed, transformed or built upon. (https://creativecommons.org/licenses/by-nc-nd/4.0/).

Thieme Medical and Scientific Publishers Private Ltd. A-12, Second Floor, Sector -2, NOIDA -201301, India 
along with the TMJ play a vital role in mandibular motion and redistribution of stress generated by activities such as chewing, swallowing, and speaking. ${ }^{2}$

The volume and size of the TMJ may be associated with the final dimensions of the mandibular arch and the relationship between the mandibular and maxillary arches. Hence, the evaluation and the assessment of the condylar volume and its morphology are of utmost importance. ${ }^{3}$

Presently, there is no objective criterion for the ideal position of the mandibular condyle within the glenoid fossa in a given population. When the posterior joint space width is less than the anterior joint space, the condyle is retruded, and it is protruded when the anterior joint space is greater. ${ }^{4}$

A markedly eccentric condylar positioning leading to alterations in joint spaces may represent an abnormality and may be a matter of concern. The pronounced anterior condylar positioning may suggest juvenile rheumatoid arthritis, and the posterior positioning of condyle may indicate the presence of disk displacement. A superior condyle placement or obliterated joint space can indicate the loss of intervening soft tissue components, disc displacement, or perforation of the joint, whereas inferior condyle placement or widened joint space may suggest the presence of blood or fluid within the joint space. ${ }^{5}$ Disc displacement, perforation, deformation, or osteoarthritis may cause narrowing of joint space. Joint space is known to be altered with condylar rotation, change in vertical dimension, condylar displacement, articular surfaces remodeling, adaptive remodeling in TMJ, neuromuscular and/or skeletal adaptive changes with orthodontic treatment or with extraction and nonextraction orthodontics. ${ }^{6}$

In the past, TMJ has been analyzed typically via twodimensional (2D) images. Conventional radiographs such as transcranial, transpharyngeal, and panoramic radiographs may be sufficient in certain cases, but they have their own limitations. ${ }^{4}$ Hence, the need for advanced modalities of imaging such as computed tomography (CT), magnetic resonance imaging (MRI), cone beam computed tomography (CBCT), and arthrography. ${ }^{7}$ The recent advancements in three-dimensional (3D) technology, especially in the field of $\mathrm{CBCT}$, have enabled us to analyze the TMJ in a more comprehensive manner than ever before. ${ }^{8}$

The aim of this research was to use CBCT imaging modality to evaluate the morphology of the mandibular condyle and glenoid fossa and document any morphological variations. Results of the previous research pertaining to the role of condylar position within the fossa among the adult population remain inconclusive. In relation to the position of the disc in adolescents, the literature is deficient in assessing the joint space area. The data obtained may provide valuable information on the anatomy of the developing TMJ and the joint spaces in adolescents with normal and altered disc positions when compared with their adult counterparts.

\section{Materials and Methods}

A retrospective study was conducted in our department. Ethical clearance for the study was obtained from the institutional ethical committee (Protocol ref. no 17130).
Large field of view (FOV) CBCT scans $(200 \times 170 \mathrm{~mm})$ of 119 patients in the age group of 18 to 50 years from September 2017 to September 2019 were obtained from the archives and assessed. Planmeca ProMax 3D mid (Planmeca, Helsinki, Finland) machine was used for capturing the CBCT scans in the occlusal state with the scan parameters being $90 \mathrm{kV}$ and $8 \mathrm{~mA}$ with a slice thickness of $0.4 \mathrm{~mm}$. The scans were analyzed using Romexis software version 4.6.2. Scans with a clear resolution and adequate coverage displaying bilateral TMJs, large FOV CBCT scans, and scans of individuals who are 18 years and above were included for the study. Scans of subjects with gross facial asymmetry, deformities, such as condylar hyperplasia/hypoplasia, history of previous orthognathic surgery, fracture of the condyle; patients with systemic diseases such as rheumatoid arthritis, Sjogren syndrome, reactive arthritis, and systemic lupus erythematous; patients presenting with a history of any tumor or growth in the orofacial region that can influence the morphology of the condyle or affect the dimensions of the joint spaces, scans with inadequate clarity or resolution; and patients below 18 years of age were excluded from the study.

\section{Procedure}

- Metric evaluation of morphology of the mandibular condyle and glenoid fossa, including its condylar height, width, length, roof of glenoid fossa (RGF) thickness, anterior, posterior, and superior joint space, and volume of the condyle were recorded as described by Al-koshab et al. ${ }^{2}$

- A 2D sagittal slice with a clear appearance of the condyle and glenoid fossa was selected. The superior-most portion of the mandibular condyle was denoted as superior mandibular condyle (SCo). Two points, one posterior and one anterior, on either side of the condyle at a distance of $4 \mathrm{~mm}$ from the SCo were selected as posterior-most mandibular condyle point (PCo) and anterior-most condylar point (ACo). From this selected sagittal slice, the condylar length was measured (-Fig. 1).

- The areas of maximum convex curvature on either side of the condyle, in the coronal section, were chosen as the medial condyle (MCo) and lateral condyle (LCo). Condylar

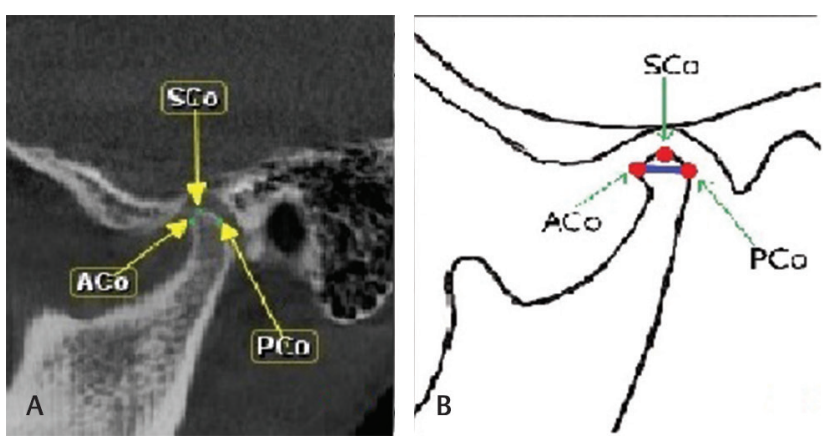

Fig. 1 (A and $\mathbf{B}$ ): Cone beam computed tomography image in sagittal view and schematic representation of the condylar length in the anteroposterior direction. ACo, anterior-most condylar point; PCo, posterior-most mandibular condyle point; SCo, superior mandibular condyle. 
width was measured as the linear distance between the mandibular lateral and medial poles (-Fig. 2).

- Height of the condyle was calculated as the perpendicular distance from SCo to a line drawn between the inferior-most point of the sigmoid notch (Inf Sig), perpendicular to the tangent of the posterior surface of the ramus in the sagittal plane ( - Fig. 3 ).

- In sagittal plane, the thickness of the glenoid fossa was defined as the distance between the outer cortical outline and the inner cortical outline ( - Fig. 4).

The linear measurements of the joint spaces between the mandibular condyle and the glenoid fossa were measured as follows:

a. A horizontal line coinciding with the superior most convex point of the glenoid fossa (superior fossa [SF]) was denoted as the true horizontal line.

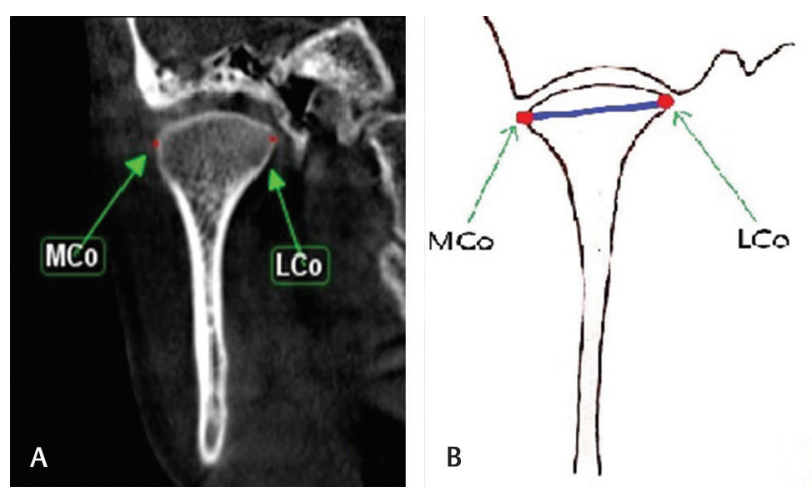

Fig. 2 ( $\mathbf{A}$ and $\mathbf{B}$ ): Cone beam computed tomography image and schematic representation of coronal view showing condylar width measurement between the two poles. LCo, maximum convex curvature on the lateral aspect of the condyle; MCo: maximum convex curvature on the mesial aspect of the condyle.
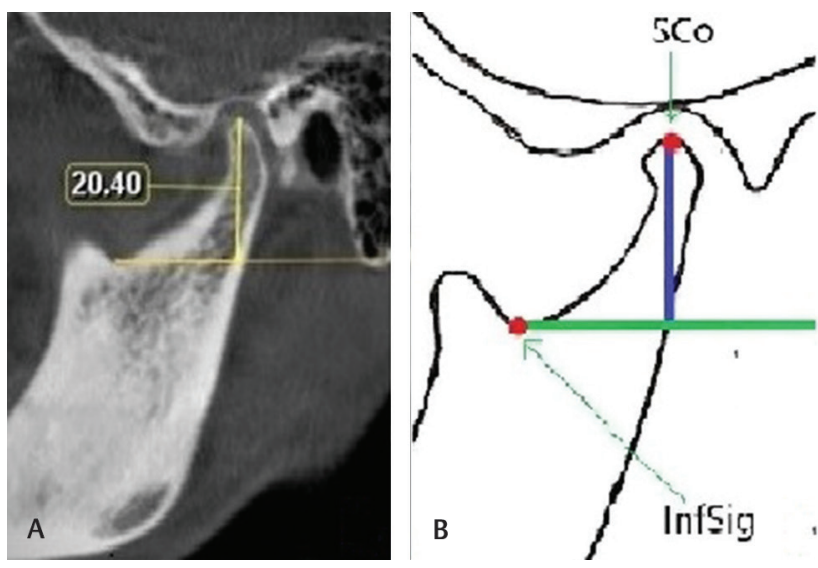

Fig. 3 (A and B): Cone beam computed tomography image and schematic representation of the sagittal view showing condylar height measurements. Inf Sig, inferior-most point of the sigmoid notch; SCo, superior mandibular condyle.

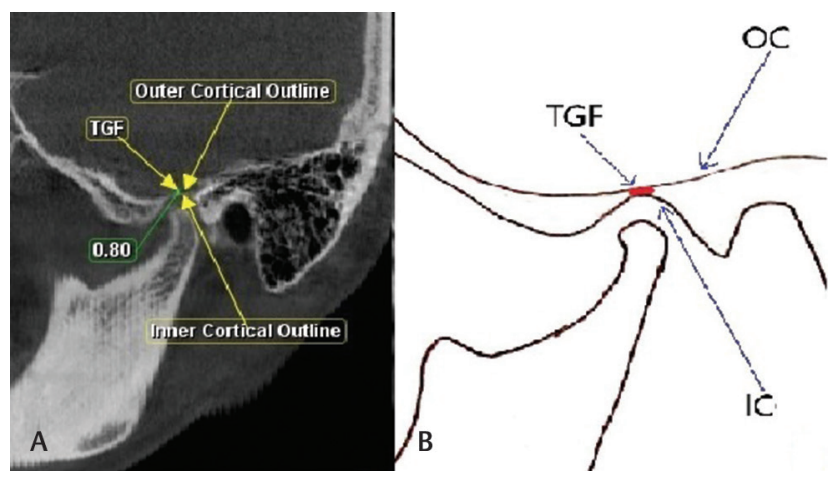

Fig. 4 ( $\mathbf{A}$ and $\mathbf{B}$ ): Cone beam computed tomography image and schematic representation of the sagittal view showing glenoid fossa roof thickness measurement between the outer and inner cortical outline of the bone. IC, inner cortical outline; OC, outer cortical outline, TGF, thickness of glenoid fossa.
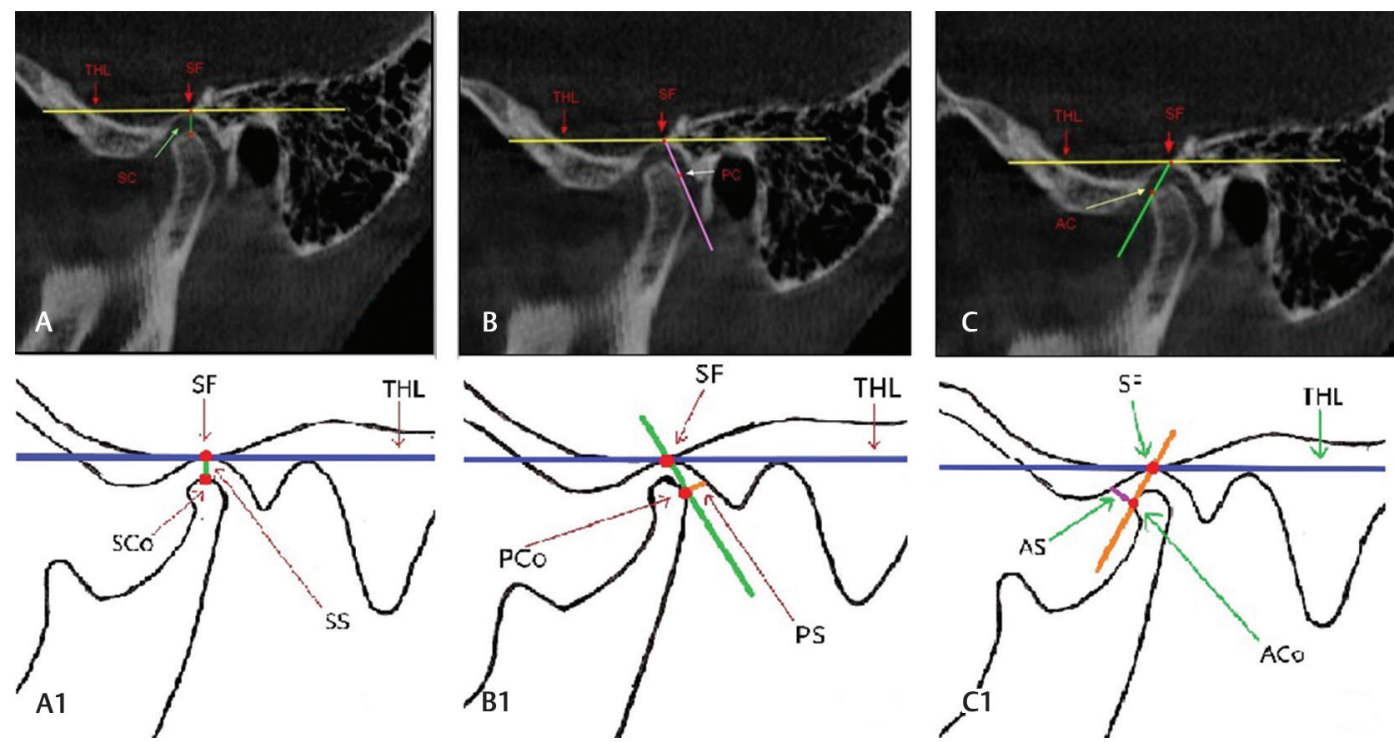

Fig. 5 (A and $\mathbf{A} \mathbf{1}$ )-Cone beam computed tomography (CBCT) image and schematic representation of the superior joint space measurements between the superior surface of the mandibular condyle and the glenoid fossa in the sagittal section; (B and B1) CBCT image and schematic representation of posterior joint space measurements between the posterior surface of the mandibular condyle and the glenoid fossa in the sagittal section. (C and C1) CBCT image and schematic representation of anterior joint space measurements between the anterior surface of the mandibular condyle and the glenoid fossa in the sagittal section. ACo, anterior-most condylar point; AS, anterior joint space; PCo, posterior-most mandibular condyle point; PS, posterior joint space; SCo. superior mandibular condyle, SS, superior joint space; THL, true horizontal line. 
b. The distance between the SCo to the SF was measured as superior joint space.

c. A tangential line connecting the most convex anterior and posterior parts of the condyle to the SF was drawn to determine the points coinciding with the anterior and posterior spaces. A perpendicular line was drawn to the tangential line to determine the distance of the glenoid fossa to the anterior condyle and the posterior condyle and was measured as the anterior space and posterior space, respectively ( - Fig. 5).

According to Al-koshab et al, ${ }^{2}$ the upper extent of the mandibular condylar head was determined in the axial view on the appearance of first radiopaque point in the joint space when the axial images were scrolled from one end to the other of the joint space (window-windowing). The lower extent was determined upon the disappearance of the radiopaque sigmoid notch. Following this procedure, the "region growing tool" in the Romexis software was used to determine the boundaries of the condyle in the axial section and a volume of the selected region was created. This corresponded to the volume of the condyle ( - Fig. 6 ).

All the measurements were performed by a maxillofacial radiologist with an experience of more than 10 years. Measurements were repeated for 20 CBCT scans by the maxillofacial radiologist after 2 weeks. The intraobserver reliability assessed through the intraclass correlation coefficient was 0.90 .

Collected data were analyzed using the Statistical Package for the Social Sciences (SPSS) software 20.0 for windows (SPSS Inc., Chicago, Illinois, United States). Correlation of the various parameters with age and gender was done using independent $t$-test. The comparisons of the values of right and left side were done using paired $t$-test. A $p$-value of $<0.05$ was considered statistically significant.

\section{Results}

A total of 119 patients including 60 females and 59 males were evaluated in the study. The length, width, height, volume, RGF thickness, and metric evaluation of all the joint spaces were performed on both the sides in each patient. Evaluation of each of the parameters between males and females was done using independent $t$-test. On comparison of each of the parameters between the two genders, there was significant variation seen with respect to the length, width, height, volume of the condyle, RGF thickness, posterior and the superior joint spaces, with a $p$-value $<0.001$. The length, width, height, volume, RGF thickness, and all the joint spaces were significantly larger in males compared with females. According to the results, only the value of left anterior joint space did not show any significant variation between males and females, even though it was higher in males (-Table $\mathbf{1}$ ).

Among the 119 patients, all the parameters were analyzed and compared between the two sides. The comparison of right and left values was done using a paired $t$-test. In males, the width of the condyle on the left side was significantly greater than that on the right with $p<0.001$. According to the results, in both males and females, the volume of the condyle was
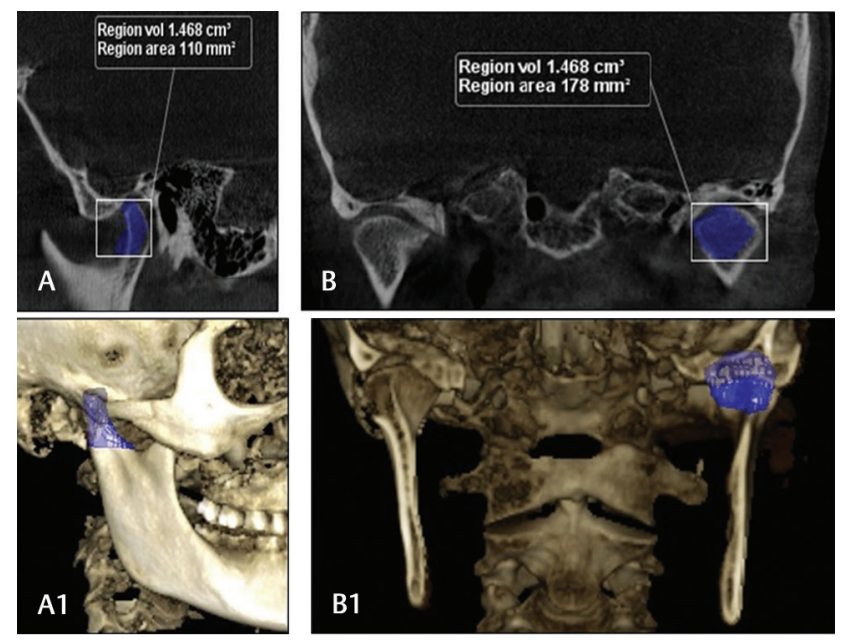

Fig. 6 (A and $\mathbf{A} \mathbf{1})$ Cone beam computed tomography (CBCT) image and three-dimensional (3D) reconstructed image in the sagittal view showing the measured volume of condyle; (B and B1) CBCT image and $3 \mathrm{D}$ reconstructed image in the coronal view showing the measured volume of condyle.

significantly larger on the left side compared with the right with $p<0.001$. It was also noted that the thickness of RGF in females was significantly more on the left side as compared with the right with $p<0.001$. Without taking gender into consideration, among the 119 patients, on comparison, there was significant difference in the width, joint spaces (anterior, superior and posterior) and the volume of the condyle between the right and left side with a $p$-value $<0.001$ ( - Table 2 ).

The study consisted of 119 patients and the median age of the sample was taken as 25 years. Patients were categorized as those above 25 years of age and those below it. There were 59 males, of which 33 were above the age of 25 years and 26 were below the age of 35 . Likewise, there were 60 females, of which 24 being less than 25 years of age and 36 were above the age of 25 years. Evaluation of each of the parameters with age (above 25 years of age and below 25 years of age) was done using a paired $t$-test. In males, only the length of the condyle on both the sides and the anterior space on the right side showed significant variation with age with a $p$-value $<0.001$ (- Table 3). In females, the length, width, height, joint spaces, and volume of the condyle showed significant variation with age with a $p$-value $<0.001$ ( $~$ Table 4 ).

\section{Discussion}

The accuracy of CBCT for linear measurements was confirmed in various studies by Lascala et al, ${ }^{8}$ Soumalainen et al, ${ }^{9}$ and Kobayashi et $\mathrm{al}^{10}{ }^{10}$ Furthermore, Lascala et al ${ }^{8}$ stated that $\mathrm{CBCT}$ images underestimate the actual distances on the base of the skull between different points and are accurate for linear measurements of these structures. Soumalainen et $\mathrm{al}^{9}$ stated that the error in linear measurement and evaluation using $\mathrm{CBCT}$ technique was lower than that of multislice CT.

Dalili et al, ${ }^{11}$ in their study, concluded that males have larger linear measurements of joint spaces when compared with females, especially the posterior and the superior spaces. 
Table 1 Comparison of various parameters of the TMJ between genders

\begin{tabular}{|c|c|c|c|c|}
\hline Parameters (in mm) & Male $(n=59)$ & Female $(n=60)$ & $t$ & $p$-Value ${ }^{a}$ \\
\hline Right. length (PCo-ACo) & $8.078 \pm 0.508$ & $6.465+0.283$ & 21.37 & $<0.001$ \\
\hline Right width (MCo-LCo) & $18.802 \pm 0.885$ & $16.65+0.627$ & 15.28 & $<0.001$ \\
\hline Right height (SCo- InfSig) & $19.07 \pm 0.766$ & $17.176+0.451$ & 16.4 & $<0.001$ \\
\hline Right anterior space & $2.252 \pm 0.323$ & $1.756+0.205$ & 9.989 & $<0.001$ \\
\hline Right superior space & $3.005 \pm 0.246$ & $2.064+0.218$ & 22.1 & $<0.001$ \\
\hline Right posterior space & $2.707 \pm 0.509$ & $1.986 \pm 0.171$ & 10.31 & $<0.001$ \\
\hline Right volume $\left(\mathrm{mm}^{3}\right)$ & $2914.698 \pm 408.384$ & $1853.335+178.458$ & 18.32 & $<0.001$ \\
\hline Right RGF & $1.472 \pm 0.093$ & $1.343+0.128$ & 6.287 & 0.001 \\
\hline Left length (PCo-ACo) & $8.086 \pm 0.491$ & B. $489+0.321$ & 20.96 & $<0.001$ \\
\hline Left width (MCo-LCo) & $18.918 \pm 0.923$ & $16.693+0.587$ & 15.67 & $<0.001$ \\
\hline Left height (SCo-InfSig) & $18.929 \pm 2.393$ & $17.223+0.426$ & 5.394 & $<0.001$ \\
\hline Left AS & $2.289 \pm 0.314$ & $2.828+4.094$ & -1.018 & 0.313 \\
\hline Left SS & $3.114 \pm 0.359$ & $2.108+0.246$ & 17.82 & $<0.001$ \\
\hline Left PS & $2.727 \pm 0.48$ & $2.023+0.203$ & 10.39 & $<0.001$ \\
\hline Left volume $\left(\mathrm{mm}^{3}\right)$ & $2957.317 \pm 415.355$ & $1870.162+183.148$ & 18.42 & $<0.001$ \\
\hline Left RGF & $1.447 \pm 0.19$ & $1.35+0.128$ & 3.285 & 0.001 \\
\hline
\end{tabular}

Abbreviations: ACo, anterior-most condylar point; AS, anterior joint space; Inf Sig, inferior-most point of the sigmoid notch; LCo, lateral condyle; MCo, medial condyle; PCo, posterior-most mandibular condyle point; PS, posterior joint space; RGF, glenoid fossa roof thickness; SD, standard deviation; SS, superior joint space; TM], temporomandibular joint.

Statistical test: Independent $t$-test.

andicates statistically significant value.

This was in accordance with the results of our study, wherein a significant difference was observed between the two genders when linear dimensions of posterior and superior joint spaces were compared. The larger joint spaces in males could be due to the thicknesses of soft tissues of the TMJ compartment, as described by Lubsen et al. ${ }^{12}$ Another possible explanation could be due to the difference in the overall size of temporal fossa and mandibular condyle in males as a result of sexual dimorphism as described by Hinton. ${ }^{13}$

In our study, linear measurements of the joint spaces displayed significant differences with age. The sample included 119 patients with the age ranging from 18 to 44 years. There was a progressive decrease in the linear measurements of anterior, posterior, and superior space with increasing age. This was in agreement with the results of a study by Alexiou et $\mathrm{al}^{14}$ who reported of a decrease in the linear measurement of the joint spaces with increasing age. This negative correlation could be due to an increase in the prevalence of bony changes with advancing age. According to Alexiou et $\mathrm{al}_{1}{ }^{14}$ degenerative changes in the condyle are generally more obvious in patients over 40 years of age.

In our study, volume of the condyle was greater in males and was significant and this was in agreement with the results of a study by Tadej et al. ${ }^{15} \mathrm{He}$ found that the overall size of the mandibular condyle was significantly larger in males as a result of sexual dimorphism $1 .^{5}$

On comparison of the measurements of the posterior and superior space among left and right sides, a significant difference was observed in both the genders. This was in agreement with the results by Cohlmia et al, ${ }^{16}$ who investigated in detail about the relationship of mandibular condyle and glenoid fossa in individuals with different types of malocclusion and skeletal patterns. He found a significant difference in values between the right and left sides in the posterior and superior joint spaces. This asymmetry observed in our research in the condylar location between the left and right sides of glenoid fossa could be explained by the presence of a preferred side for mastication in patients with malocclusion as stated by Blaschke and Blaschke. ${ }^{17}$

Wang et $\mathrm{al}^{18}$ and Rodrigues et $\mathrm{al},{ }^{19}$ in a detailed analysis of the thickness of the RGF on right and left sides in an individual, concluded that there was significant variation between the two sides. This was in agreement with our research wherein we found a difference in the thickness of the RGF between the two sides and was significant in females, with the left side being significantly larger. This asymmetry may be associated with normal cranial base asymmetries as reported by Kijima et al..$^{20}$ The condyle morphology responds to the changing demands of the masticatory process, including those arising from teeth loss and occlusal wear. The condyle morphology and RGF thickness are therefore influenced by an external stimulus that influences the difference between the right and left sides. ${ }^{20}$

In our study, the volume of condyle was significantly increased on the left side in both males and females. This was in accordance with the results of a study by Tecco et al. ${ }^{21}$ 
Table 2 Correlation of measurements between right and left side

\begin{tabular}{|c|c|c|c|c|c|c|}
\hline Gender & Parameter & $\begin{array}{l}\text { Mean } \pm \text { SD }(\mathrm{mm}) \\
\text { right }\end{array}$ & $\begin{array}{l}\text { Mean } \pm \text { SD }(\mathrm{mm}) \\
\text { left }\end{array}$ & $\begin{array}{l}\text { Mean } \\
\text { difference } \pm \text { SD }\end{array}$ & $t$ & $p$-Value \\
\hline \multirow[t]{8}{*}{ Male $(n=59)$} & Length. & $8.08 \pm 0.51$ & $8.09+0.49$ & $0.01 \pm 0.09$ & 0.68 & 0.497 \\
\hline & Width & $18.8 \pm 0.89$ & $18.92 \pm 0.92$ & $0.12 \pm 0.13$ & 6.79 & $<0.001^{\mathrm{a}}$ \\
\hline & Height & $19.07 \pm 0.77$ & $18.93 \pm 2.39$ & $0.14 \pm 2.2$ & 0.49 & 0.624 \\
\hline & AS & $2.25 \pm 0.32$ & $2.29 \pm 0.31$ & $0.04 \pm 0.17$ & 1.68 & 0.098 \\
\hline & SS & $3 \pm 0.25$ & $3.11 \pm 0.36$ & $0.11 \pm 0.28$ & 2.95 & $0.005^{\mathrm{a}}$ \\
\hline & PS & $2.71 \pm 0.51$ & $2.73 \pm 0.48$ & $0.02 \pm 0.06$ & 2.47 & $0.017^{\mathrm{a}}$ \\
\hline & Volume $\left(\mathrm{mm}^{3}\right)$ & $2914.7 \pm 408.38$ & $2957.32 \pm 415.36$ & $42.62 \pm 41.01$ & 7.98 & $<0.001^{\mathrm{a}}$ \\
\hline & RGF & $1.47 \pm 0.09$ & $1.45 \pm 0.19$ & $0.02 \pm 0.19$ & 1.01 & 0.319 \\
\hline \multirow[t]{8}{*}{ Female $(n=60)$} & Length. & $6.47 \pm 0.28$ & $6.49 \pm 0.32$ & $0.02 \pm 0.07$ & 2.86 & $0.006^{\mathrm{a}}$ \\
\hline & Width & $16.65 \pm 0.63$ & $16.69 \pm 0.59$ & $0.04 \pm 0.15$ & 2.15 & $0.035^{\mathrm{a}}$ \\
\hline & Height & $17.18 \pm 0.45$ & $17.22 \pm 0.43$ & $0.05 \pm 0.14$ & 2.49 & $0.015^{\mathrm{a}}$ \\
\hline & AS & $1.76 \pm 0.21$ & $2.83 \pm 4.09$ & $1.07 \pm 4.08$ & 2.04 & $0.046^{\mathrm{a}}$ \\
\hline & SS & $2.06 \pm 0.22$ & $2.11 \pm 0.25$ & $0.04+0.09$ & 3.59 & $0.001^{\mathrm{a}}$ \\
\hline & PS & $1.99 \pm 0.17$ & $2.02 \pm 0.2$ & $0.04+0.09$ & 2.98 & $0.004^{\mathrm{a}}$ \\
\hline & Volume $\left(\mathrm{mm}^{3}\right)$ & $1853.34 \pm 178.46$ & $1870.16 \pm 183.15$ & $16.83+32.94$ & 3.96 & $<0.001^{\mathrm{a}}$ \\
\hline & RGF & $1.34 \pm 0.13$ & $1.36 \pm 0.13$ & $0.01 \pm 0.03$ & 2.05 & $<0.001^{\mathrm{a}}$ \\
\hline \multirow[t]{8}{*}{ Total $(n=119)$} & Length. & $7.26 \pm 0.91$ & $7.28 \pm 0.9$ & $0.02+0.08$ & 2.25 & $0.026^{\mathrm{a}}$ \\
\hline & Width & $17.72 \pm 1.32$ & $17.8 \pm 1.36$ & $0.08+0.15$ & 5.87 & $<0.001^{\mathrm{a}}$ \\
\hline & Height & $18.12 \pm 1.14$ & $18.07 \pm 1.91$ & $0.05+1.55$ & 0.33 & 0.744 \\
\hline & AS & $2 \pm 0.37$ & $2.56 \pm 2.92$ & $0.56+2.93$ & 2.08 & $0.04^{\mathrm{a}}$ \\
\hline & SS & $2.53 \pm 0.53$ & $2.61 \pm 0.59$ & $0.08+0.21$ & 3.91 & $<0.001^{\mathrm{a}}$ \\
\hline & PS & $2.34 \pm 0.52$ & $2.37 \pm 0.51$ & $0.03+0.08$ & 3.84 & $<0.001^{\mathrm{a}}$ \\
\hline & Volume $\left(\mathrm{mm}^{3}\right)$ & $2379.56 \pm 617.97$ & $2409.17 \pm 632.08$ & $29.61 \pm 39.2$ & 8.24 & $<0.001^{\mathrm{a}}$ \\
\hline & RGF & $1.41 \pm 0.13$ & $1.4 \pm 0.17$ & $0.01 \pm 0.13$ & 0.72 & 0.472 \\
\hline
\end{tabular}

Abbreviations: AS, anterior joint space; PS, posterior joint space; RGF, roof of glenoid fossa; SS, superior joint space.

Statistical test: Paired $t$-test.

andicates statistically significant value.

Table 3 Correlation of the various parameters of TMJ morphology with age among male subjects

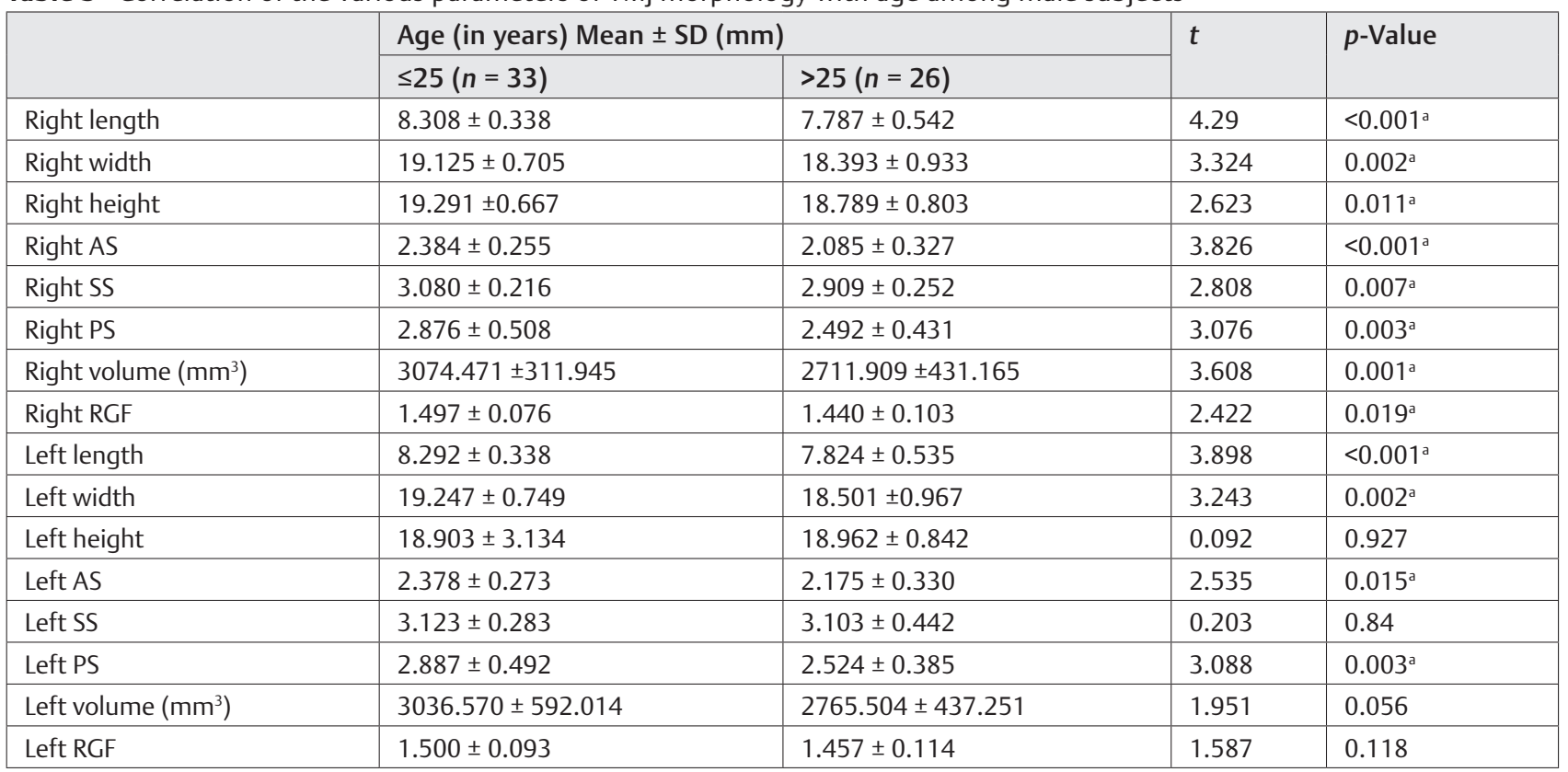

Abbreviations: AS, anterior joint space; PS, posterior joint space; RGF, roof of glenoid fossa; SD, standard deviation; SS, superior joint space; TM], temporomandibular joint.

Statistical test: Independent $t$-test B.

andicates statistically significant value. 
Table 4 Correlation of the various parameters of TMJ morphology with age among female subjects

\begin{tabular}{|c|c|c|c|c|}
\hline \multirow[t]{2}{*}{ Parameters (in mm) } & \multicolumn{2}{|c|}{ Age in Years ( Mean \pm Std Deviation $(\mathrm{mm})$ ) } & \multirow[t]{2}{*}{$t$} & \multirow[t]{2}{*}{$p$-Value } \\
\hline & $\leq 25(n=24)$ & $>25(n=36)$ & & \\
\hline Right length & $6.754 \pm 0.172$ & $6.272 \pm 0.141$ & 11.89 & $<0.001^{*}$ \\
\hline Right width & $17.126 \pm 0.621$ & $16.333 \pm 0.387$ & 5.573 & $<0.001 *$ \\
\hline Right height & $17.328 \pm 0.549$ & $17.075 \pm 0.344$ & 2.015 & 0.052 \\
\hline Right AS & $1.871 \pm 0.257$ & $1.679 \pm 0.112$ & 3.447 & $0.002^{*}$ \\
\hline Right SS & $2.255 \pm 0.218$ & $1.937 \pm 0.086$ & 6.79 & $<0.001^{*}$ \\
\hline Right PS & $2.094 \pm 0.210$ & $1.914 \pm 0.085$ & 3.98 & $<0.001^{*}$ \\
\hline Right Volume $\left(\mathrm{mm}^{3}\right)$ & $2008.762 \pm 172.595$ & $1749.718 \pm 80.629$ & 6.87 & $<0.001^{*}$ \\
\hline Right RGF & $1.333 \pm 0.174$ & $1.350 \pm 0.088$ & -0.435 & 0.667 \\
\hline Left length & $6.804 \pm 0.222$ & $6.279 \pm 0.167$ & 10.45 & $<0.001^{*}$ \\
\hline Left width & $17.135 \pm 0.559$ & $16.398 \pm 0.389$ & 5.612 & $<0.001^{*}$ \\
\hline Left height & $17.331 \pm 0.479$ & $17.150 \pm 0.377$ & 1.633 & 0.108 \\
\hline Left AS & $4.553 \pm 6.149$ & $1.679 \pm 0.094$ & 2.289 & $0.032^{*}$ \\
\hline Left SS & $2.306 \pm 0.242$ & $1.975 \pm 0.136$ & 6.778 & $<0.001^{*}$ \\
\hline Left PS & $2.149 \pm 0.240$ & $1.939 \pm 0.117$ & 3.985 & $<0.001^{*}$ \\
\hline Left Volume $\left(\mathrm{mm}^{3}\right)$ & $2025.015 \pm 173.457$ & $1766.926 \pm 96.786$ & 6.633 & $<0.001^{*}$ \\
\hline Left RGF & $1.342 \pm 0.184$ & $1.356 \pm 0.073$ & -0.352 & 0.728 \\
\hline
\end{tabular}

Abbreviations: AS: Anterior Joint Space; PS: Posterior Joint Space; SS: Superior Joint Space; RGF: roof of glenoid fossa.

Statistical test: Independent $t$-test B.

"Indicates statistically significant value.

The asymmetry seen in our analysis between the two sides could be due to the presence of a preferred side of chewing among subjects with malocclusion, contributing to an altered morphology and volume. ${ }^{22}$ The volume of the condyle also varies with the type of malocclusion. This was explained by Moss's Functional matrix theory, which stated that the growth of each skeletal unit in the maxillofacial surface is defined based on the functional requirement of the corresponding tissue. ${ }^{22}$

In the present study, the volume of the condyle decreased with increasing age and was significant in females. This was in accordance with the results of Takayama et $\mathrm{al}^{23}$ who reported of a decrease in the volume of the condyle with age, in both males and females. He concluded that the volumetric and morphologic changes are more common in TMJ with increasing age, and age is the paramount factor that determines the degree of remodeling. As the adaptive and degenerative changes appear in the TMJ over a long period of time, the volume of the condyle decreases with increasing age. ${ }^{23}$

The length, width, and height of the mandibular condyle among males were significantly greater on both the sides. This was in accordance with the results of Tadej et $\mathrm{al}^{15}$ who evaluated the mediolateral width and height of condyle in children with malocclusion. According to Enomoto et $\mathrm{al},{ }^{24}$ the type of diet and parafunctional habits leads to discrepancies in measurements between the two sides.

In our study, the length and width of the condyle in both males and females were significantly increased in the below 25 years age group. This result was in agreement with the results achieved by Chaurasia et $\mathrm{al}^{25}$ who observed significant differences in the length and width of the condyle with age, in both males and females. He concluded that the condylar height and width are maximum in the 20 to 30 age group and gradually decrease with increasing age.

According to $\mathrm{Kim},{ }^{26}$ mean joint space estimated was $2.77 \pm 0.51 \mathrm{~mm}$ anteriorly, $3.57 \pm 0.63 \mathrm{~mm}$ superiorly, and $2.41 \pm 0.41 \mathrm{~mm}$ posterior to the mandibular condyle. The mean joint space was larger in males. The noncentralization of the mandibular condyles has been reported to be a striking feature in different type of skeletal relationships and malocclusions. Patients having skeletal and dental Class III malocclusions showed significantly more anteriorly positioned condyle. The significant difference among the right and left sides observed in our study suggested that the right side has a more posteriorly placed fossa, narrow glenoid fossa width, and greater inclination of the anterior wall, and the left condyle has increased condylar width and vertical inclination. These details are paramount prior to any orthodontic treatment or orthognathic surgery.

\section{Conclusion}

In our study, there was a significant variation in the linear condylar measurements of width and volume between the left and right side that emphasize the fact that each condyle should be evaluated and assessed as a separate entity prior to any treatment. In surgical procedures, such as orthognathic surgery and orthodontic management, these anatomical dimensional variabilities can be significant.

These parameters also displayed a significant negative association with age. The findings of our study also revealed variations in linear measurements in both the genders. Among females, there were significant differences seen 
on comparing the thickness of RGF between the two sides. Males generally displayed a larger condylar volume and size.

A detailed CBCT analysis of TMJ would aid in precisely evaluating the morphological variations in different maxillofacial alignments and in analyzing the positional and morphological TMJ changes post extraction and nonextraction orthodontic treatment, facemask therapy, and functional appliances (orthopaedic) and in orthognathic surgical procedures. For future research, the data from the present study could be used as a guideline for various parameters of the TMJ, although a larger cohort study and a detailed comparison with other imaging modalities including CT and MRI especially in the evaluation of the joint spaces are desirable and are $\mathrm{a}$ few of the limitations in the present study.

CBCT can be a valuable diagnostic aid in the evaluation of various morphometric analysis of the TMJ, its joint spaces, and condylar volume in different planes and thus can be a useful predictor in the assessment of treatment outcomes in disorders affecting the TMJ.

\section{Financial Support and Sponsorship \\ Nil.}

\section{Conflicts of Interest}

There are no conflicts of interest.

\section{References}

1 Pandis N, Karpac J, Trevino R, Williams B. A radiographic study of condyle position at various depths of cut in dry skulls with axially corrected lateral tomograms. Am J Orthod Dentofacial Orthop 1991;100(2):116-122

2 Al-koshab M, Nambiar P, John J. Assessment of condyle and glenoid fossa morphology using CBCT in South-East Asians. PLoS One 2015;10(3):e0121682

3 Ejima K, Schulze D, Stippig A, Matsumoto K, Rottke D, Honda K. Relationship between the thickness of the roof of glenoid fossa, condyle morphology and remaining teeth in asymptomatic European patients based on cone beam CT data sets. Dentomaxillofac Radiol 2013; 42(3):9092941010.1259/dmfr/90929410

4 Nancy A, Tencate's Oral Histology Development, Structure and Functions. Temporomandibular Joint. 8th edition. Ch. 13. Missouri: Elsevier Mosby Publishers; 2013400

5 Major P, Kamelchuk L, Nebbe B, Petrikowski G, Glover K. Condyle displacement associated with premolar extraction and nonextraction orthodontic treatment of Class I malocclusion. Am J Orthod Dentofacial Orthop 1997;112(4):435-440

6 Marques AP, Perrella A, Arita ES, Pereira MF, Cavalcanti MdeG. Assessment of simulated mandibular condyle bone lesions by cone beam computed tomography. Braz Oral Res 2010;24(4):467-474

7 Miloglu O, Yilmaz AB, Yildirim E, Akgul HM. Pneumatization of the articular eminence on cone beam computed tomography: prevalence, characteristics and a review of the literature. Dentomaxillofac Radiol 2011;40(2):110-114

8 Lascala CA, Panella J, Marques MM. Analysis of the accuracy of linear measurements obtained by cone beam computed tomography (CBCT-NewTom) Dentomaxillofac Radiol 2004;33(5):291-294

9 Suomalainen A, Vehmas T, Kortesniemi M, Robinson S, Peltola J. Accuracy of linear measurements using dental cone beam and conventional multislice computed tomography. Dentomaxillofac Radiol 2008;37(1):10-17
10 Kobayashi K, Shimoda S, Nakagawa Y, Yamamoto A. Accuracy in measurement of distance using limited cone-beam computerized tomography. Int J Oral Maxillofac Implants 2004;19(2):228-231

11 Dalili Z, Khaki N, Kia SJ, Salamat F. Assessing joint space and condylar position in the people with normal function of temporomandibular joint with cone-beam computed tomography. Dent Res J (Isfahan) 2012;9(5):607-612

12 Lubsen CC, Hansson TL, Nordström BB, Solberg WK. Histomorphometry of age and sex changes in mandibular condyles of young human adults. Arch Oral Biol 1987; 32(10):729-733

13 Hinton RJ. Relationships between mandibular joint size and craniofacial size in human groups. Arch Oral Biol 1983;28(1):37-43

14 Alexiou K, Stamatakis H, Tsiklakis K. Evaluation of the severity of temporomandibular joint osteoarthritic changes related to age using cone beam computed tomography. Dentomaxillofac Radiol 2009;38(3):141-147

15 Tadej G, Engstrom C, Borrman H, Christiansen EL. Mandibular condyle morphology in relation to malocclusions in children. Angle Orthod 1989;59(3):187-194

16 Cohlmia JT, Ghosh J, Sinha PK, Nanda RS, Currier GF. Tomographic assessment of temporomandibular joints in patients with malocclusion. Angle Orthod 1996;66(1):27-35

17 Blaschke DD, Blaschke TJ. A method for quantitatively determining temporomandibular joint bony relationships. J Dent Res 1981;60(1):35-43

18 Wang RY, Ma XC, Zhang WL, Liu DG. [Investigation of temporomandibular joint space of healthy adults by using cone beam computed tomography]. Beijing Da Xue Xue Bao 2007;39(5):503-506

19 Rodrigues AF, Fraga MR, Vitral RW. Computed tomography evaluation of the temporomandibular joint in Class II Division 1 and Class III malocclusion patients: condylar symmetry and condyle-fossa relationship. Am J Orthod Dentofacial Orthop 2009;136(2):199-206

20 Kijima N, Honda K, Kuroki Y, Sakabe J, Ejima K, Nakajima I. Relationship between patient characteristics, mandibular head morphology and thickness of the roof of the glenoid fossa in symptomatic temporomandibular joints. Dentomaxillofac Radiol 2007;36(5):277-281

21 Tecco S, Saccucci M, Nucera R, et al. Condylar volume and surface in Caucasian young adult subjects. BMC Med Imaging 2010;10:28

22 Mostafavi M, Vahdat S, Javadian L, Ghaznavi A. Analysis of condylar volume in relation to craniofacial morphology using cone beam computed tomography. J Contemporary Med Sci 2018;4:202-206

23 Takayama Y, Miura E, Yuasa M, Kobayashi K, Hosoi T. Comparison of occlusal condition and prevalence of bone change in the condyle of patients with and without temporomandibular disorders. Oral Surg Oral Med Oral Pathol Oral Radiol Endod 2008;105(1):104-112

24 Enomoto A, Watahiki J, Yamaguchi T, Irie T, Tachikawa T, Maki K. Effects of mastication on mandibular growth evaluated by microcomputed tomography. EurJOrthod 2010;32(1):66-70

25 Chaurasia A, Katheriya G, Khan N. Morphometric analysis of articular eminence of temporomandibular joint in Indian paediatric population: a CBCT study. Indian J Forensic Odontol 2017;10:91-103

26 Kim JB. Normal temporomandibular joint space. J Korean Assoc Oral Maxillofac Surg 2000;26:279-283 